\title{
Both Osteopontin-c and Osteopontin-b Splicing Isoforms Exert Pro-Tumorigenic Roles in Prostate Cancer Cells
}

\author{
Tatiana M. Tilli, ${ }^{1}$ Kivvi D. Mello, ${ }^{1}$ Luciana B. Ferreira, ${ }^{1}$ Aline R. Matos, ${ }^{1}$ \\ Maria Thereza S. Accioly, ${ }^{2}$ Paulo A.S. Faria, ${ }^{2}$ Akeila Bellahcène, ${ }^{3}$ \\ Vincent Castronovo, ${ }^{3}$ and Eel R. Gimba ${ }^{1,4 *}$ \\ 'Coordenação de Pesquisa, Programa de Medicina Experimental, Instituto Nacional de Câncer (INCa)/Programa de \\ Pós Graduação Stricto Sensu em Oncologia do INCa, Rio de Janeiro, RJ, Brazil \\ ${ }^{2}$ Divisão de Patologia, INCa, Rio de Janeiro, RJ, Brazil \\ ${ }^{3}$ Metastasis Research Laboratory, Grappe Disciplinaire de Génoprotéomique Appliquée (GIGA)-Cancer, Liège \\ University, Liège, Belgium \\ ${ }^{4}$ Departamento Interdisciplinar de Rio das Ostras (RIR), Polo Universitário de Rio das Ostras (PURO)/Universidade \\ Federal Fluminense, Rio das Ostras, RJ, Brazil
}

\begin{abstract}
BACKGROUND. Alternative splicing of the osteopontin (opn, spp1) gene generates three protein splicing isoforms (OPN-SI), designated as OPNa, OPNb, and OPNc, which have demonstrated specific roles in different tumor models. This work aims to investigate the roles of each OPN-SI in prostate cancer (PCa) progression by using in vivo and in vitro functional assays.

METHODS. The expression levels of OPN-SI in prostate cell lines were analyzed by qRT-PCR. PC-3 was stably transfected with expression vectors containing OPNa, OPNb, and OPNc, as well as empty vector controls. PC-3 cells overexpressing each construct were analyzed for in vivo tumor growth and in relation to different aspects mimicking tumor progression, such as cell proliferation, migration, invasion, and soft agar colony formation.

RESULTS. OPN-SI are overexpressed in PCa as compared to non-tumoral prostate cell lines. $\mathrm{OPNc}$ and $\mathrm{OPNb}$ overexpressing cells significantly activated enhanced xenograft tumor growth and PC-3 proliferation, migration, invasion, and soft agar colony formation, as well as the expression of MMP-2, MMP-9, and VEGF. These isoforms also support sustained proliferative survival. We found that both OPNc and OPNb pro-tumorigenic roles are mainly mediated through PI3K signaling. Inhibition of this pathway by using LY294002 specifically inhibited tumor progression features evoked by OPNc and OPNb overexpression.

CONCLUSIONS. Our data provide evidence that both OPNc and OPNb splicing isoforms promote distinct aspects of PCa progression by inducing PI3K signaling. These data give support to strategies aiming to downregulate OPNc and OPNb expression as an approach to inhibit PCa progression. Prostate (C) 2012 Wiley Periodicals, Inc.
\end{abstract}

KEY WORDS: osteopontin; splicing isoforms; alternative splicing; prostate cancer

Additional supporting information may be found in the online version of this article.

Grant sponsor: Swiss Bridge Foundation; Grant number: E-26/ 111.496/2008; Grant sponsor: FAPERJ; Grant numbers: 103.124/2008; 110.506/2011; Grant sponsor: CNPq; Grant number: 573806/2008-0; Grant sponsor: INCT.
*Correspondence to: Dr. E.R. Gimba, Polo Universitário de Rio das Ostras-UFF/Instituto Nacional de Câncer Rua Recife s/n, Bairro Bela Vista, Rio das Ostras-RJ-Brazil. E-mail: etelgimba@id.uff.br Received 5 November 2011; Accepted 5 March 2012 DOI 10.1002/pros.22523

Published online in Wiley Online Library (wileyonlinelibrary.com). 


\section{INTRODUCTION}

Prostate cancer (PCa) comprises a heterogeneous group of tumors with a broad spectrum of pathological, molecular features and clinical behaviors. This diversity confounds the development of therapy strategies and of specific biomarkers, especially due to differences in genetic composition. An improved understanding of the molecular basis of prostate carcinogenesis and tumor progression could establish new PCa markers and also help to identify new targets for improved treatment options.

In $\mathrm{PCa}$, previous studies suggest a role for increased osteopontin (OPN, SPP1) tissue expression both in the malignant transformation of prostate epithelial cells and also as an important determinant of tumor progression and patient survival [1-5]. This glycophosphoprotein act as important autocrine and paracrine signal that affect growth and behavior of prostate carcinoma cells [2,3]. Besides, OPN has also been described as an important marker for stage, grade, and early tumor progression in PCa [6,7].

Variations in splicing enhance proteome diversity and modulate cancer-associated proteins. OPN mRNA is subject to alternative splicing, resulting in OPN splicing isoforms (OPN-SI) that are shorter than fulllength OPN [8-11]. Cancer-specific splicing variants potentially provide diagnostic, prognostic, and predictive biomarkers, in addition to potential targeted therapies [12]. Osteopontin-a (OPNa) is the full-length isoform, while osteopontin-b (OPNb) lacks exon 5 and osteopontin-c (OPNc) lacks exon 4 [9]. The roles of OPN-SI have been demonstrated to be tumor specific, although the mechanisms controlling this are currently unknown $[8-11,13]$. We recently published the first report about OPN isoforms in PCa, by demonstrating the expression pattern of each OPN-SI in $\mathrm{PCa}$ and benign prostate hyperplasia (BPH) samples [14]. We showed that OPNa, OPNb, and OPNc splice variants are overexpressed in $\mathrm{PCa}$ in relation to $\mathrm{BPH}$. Besides, we observed that among these isoforms, the OPNc variant was the most upregulated in $\mathrm{PCa}$, and outperformed the remaining isoforms and PSA serum levels in PCa diagnostic accuracy. Additionaly, immunohistochemistry analysis also demonstrated OPNc protein upregulation in PCa samples as compared to BPH tissues. OPNc protein was also strongly stained in PCa tissues presenting high Gleason Score. Although presenting this differential expression pattern, data about the roles of each OPN-SI in PCa progression are lacking, once all data regarding the functional evaluation of OPN in PCa relates to the full length isoform.

In the current study, we further identified OPN-SI transcript overexpression in $\mathrm{PCa}$ in relation to non- tumoral prostate cell lines. Based on this evidence and on our previous data [14] of a potential role for OPN-SI in PCa progression, we addressed the function of PCa-derived OPN-SI by examining the effect of their ectopic overexpression in PC-3 cells. PC-3 cells overexpressing each of the OPN-SI were evaluated for features mimicking different steps of PCa tumor progression by in vivo and in vitro approaches. Our data demonstrated by the first time that the overexpression of OPNc and OPNb increases PC-3 cell growth, sustained proliferative survival, migration, invasion, anchorage-independence, and tumor formation in vivo, suggesting a possible functional role for OPNc and $\mathrm{OPNb}$ in $\mathrm{PCa}$ progression and survival. Additionally, we demonstrated herein that these tumor promoting roles could be mediated through the activation of the Phosphatidylinositol-3 Kinase (PI3K)/ Akt signaling pathway.

\section{MATERIALS AND METHODS}

\section{Cell Culture, OPN Plasmids, and Transfection}

We used three PCa cell lines: PC-3, DU-145, and LNCaP (supplied from ATCC) and two non-tumoral prostate cell lines, RWPE-1 and PrEC, which were cultured in standard conditions. RWPE-1 and PrEC cell line were a gift from Dr. Carlos Moreno (Emory University, EUA). All cell lines were cultured in culture medium (CM) supplemented with $10 \%$ fetal bovine serum (FBS), $100 \mathrm{IU} / \mathrm{ml}$ penicillin and $100 \mathrm{mg} / \mathrm{ml}$ streptomycin in a humidified environment containing $5 \% \mathrm{CO}_{2}$ at $37^{\circ} \mathrm{C}$.

The open reading frame of OPN splice variants, $\mathrm{OPNa}, \mathrm{OPNb}$, and OPNc were cloned into pCR3.1 mammalian expression vector as previously described [9]. OPN-SI expression vectors were kindly provided by Dr. George Weber (Cincinnati University) and these DNA constructs were used for transfection into PC3. Transfections were performed using Lipofectamine $^{\mathrm{TM}} 2000$ (Invitrogen, CA). Expression plasmids were transfected into $\mathrm{PC}-3$ cells and the stably expressing cell clones were selected with $800 \mu \mathrm{g} / \mathrm{ml}$ of G418. Six OPN isoform overexpressing clones (designated OPNa1, OPNa4, OPNb2, OPNb4, OPNc1, and OPNc4) and one empty vector control clone (empty vector) were selected.

\section{Quantitative Real-Time RT-PCR (qRT-PCR)}

RNA was extracted using the RNeasy kit (Qiagen). cDNA synthesis was carried out using SuperScript II First-Strand Synthesis System for RT-PCR using oligo(dT) primer (Invitrogen) and $1 \mu \mathrm{g}$ of total RNA. Conditions for MMP-2 and MMP-9 PCR amplification by qRT-PCR were $50^{\circ} \mathrm{C}$ for $2 \mathrm{~min}, 94^{\circ} \mathrm{C}$ for $5 \mathrm{~min}$ 
followed by 40 cycles of $94^{\circ} \mathrm{C}$ for $30 \mathrm{sec}, 58^{\circ} \mathrm{C}$ for $30 \mathrm{sec}$, and $72^{\circ} \mathrm{C}$ for $90 \mathrm{sec} ; 72^{\circ} \mathrm{C}$ for $15 \mathrm{~min}$ and finally a melting curve analysis $\left(60-90^{\circ} \mathrm{C}\right.$ with a heating rate of $0.2^{\circ} \mathrm{C}$ and continuous fluorescence measurement). Conditions for VEGF PCR amplification were $50^{\circ} \mathrm{C}$ for $2 \mathrm{~min}, 94^{\circ} \mathrm{C}$ for $5 \mathrm{~min}$ followed by 40 cycles of $94^{\circ} \mathrm{C}$ for $30 \mathrm{sec}, 60^{\circ} \mathrm{C}$ for $30 \mathrm{sec}$, and $72^{\circ} \mathrm{C}$ for $90 \mathrm{sec}$; $72^{\circ} \mathrm{C}$ for $15 \mathrm{~min}$ and finally a melting curve analysis $\left(60-90^{\circ} \mathrm{C}\right.$ with a heating rate of $0.2^{\circ} \mathrm{C}$ and continuous fluorescence measurement).

The amplification of fragments corresponding to each OPN isoform, MMP-2, MMP-9, and VEGF was performed using the following oligonucleotide pairs: OPNa:5'-ATC TCC TAG CCC CAC AGA AT- $3^{\prime}$ (forward) and 5'-CAT CAG ACT GGT GAG AAT CAT C-3' (reverse); OPNb: 5'-CTC CTA GCC CCA CAG ACC CT-3' (forward) and 5'-TAT CAC CTC GGC CAT CAT ATG-3' (reverse); OPNc: 5'-CTG AGG AAA AGC AGA ATG-3' (forward) and 5'-AAT GGA GTC CTG GCT GT-3' (reverse); MMP-2: 5'-AAA ATG GAT CCT GGC TTC CC-3' (forward) and $5^{\prime}$-TAG CCA GTC GGA TTT GAT GC-3' (reverse); MMP-9: 5'-TGA CAG CGA CAA GAA GTG-3' (forward) and 5'-CAG TGA AGC GGT ACA TAG G-3' (reverse); VEGF: 5'-CTT GCC TTG CTG CTC TAC C-3' (forward) and 5'-CAC ACA GGA TGG CTT GAA G-3' (reverse). GAPDH was amplified with primers 5'- TGA CCC CTT CAT TGA CCT CA -3' (forward) and $5^{\prime}$-AGT CCT TCC ACG ATA CCA AA-3' (reverse), and Actin was amplified with primers $5^{\prime}$-TGA CCC AGA TCA TGT TTG AGA-3' (forward) and $5^{\prime}$-ACT CCA TGC CCA GGA AGG A -3' (reverse), which served as an internal control to normalize expression data and to verify integrity of the cDNA. All qRT-PCR reactions were conducted using the SYBR Green (Applied Biosystems). Relative gene expression of the target gene was calculated by using the $\Delta \Delta C T$ method.

\section{Ki-67 Staining}

The expression of Ki-67 was accomplished via immunohistochemical techniques on serial paraffin sections of xenograft tumors overexpressing each OPN-SI and controls. Tumors were fixed in $4 \%$ paraformaldehyde in $0.1 \mathrm{M}$ phosphate buffer. Samples were paraffin embedded and tissue sections were processed using standard protocols. The mouse monoclonal Ki-67 antibody (clone MIB-1, Dako) was used at a dilution of 1:50. The next incubation step employed the streptavidin-biotin-peroxidase complex (LSAB, Dako) and color development was obtained with diaminobenzidine solution (Kit DAB, Dako). The positive immunoreactions were manually counted using a $40 \times$ magnification at Olympus BH-2 microscope coupled to a digital camera.

\section{Cell Proliferation, Death, Migration, Invasion, and Soft Agar Growth Assays}

Cell proliferation was analyzed by crystal violet. Cell death analyses were conducted by using trypan blue exclusion assays. For the analysis by crystal violet and Trypan blue, wild-type PC-3, PC-3 cells transfected either with OPNa, OPNb or OPNc plasmid constructs, or pCR3.1 empty vector were plated in triplicate in 24-well microtiter plates. For these assays, $5 \times 10^{4}$ cells were plated per well. For crystal violet incorporation assays, cells were washed twice with PBS and fixed in glutharaldeyde for $10 \mathrm{~min}$, followed by staining with $0.1 \%$ crystal violet and solubilization with $0.2 \%$ Triton X-100. Microtiter plates were read on a spectrophotometer at $550 \mathrm{~nm}$. Viable and non-viable cells were counted using a Neubauer's chamber. Total number of cells/well for each cell clone group was calculated and plotted against each time point. Cell proliferation assays for OPNc depletion using anti-OPNc antibody were evaluated either in the absence or in the presence of anti-OPNc IgY antibody (Gallus Immunotech), added every other day at $4 \mu \mathrm{g} / \mathrm{ml}$. This antibody was produced by immunizing a chicken with a peptide representing the splice junction of osteopontin-c (Ac-SEEKQNAVSC$\mathrm{COOH}$ ). Specific binding to OPNc has been has been demonstrated by the providers. Proliferation assays were also performed using non-specific goat IgG and chicken IgY antibodies (Gallus Immunotech) as immunoglobulin negative controls $(4 \mu \mathrm{g} / \mathrm{ml})$. In order to test whether secreted OPN isoforms were performing the observed cell behavior, wild type PC-3 and RWPE-1 cells were incubated with conditioned media (CM) obtained from PC-3 cells transfected with $\mathrm{OPNa}, \mathrm{OPNb}, \mathrm{OPNc}$ or empty vector, and cell proliferation was measured by crystal violet incorporation. These assays were performed in serum-free conditions, in order to eliminate skewing of the results by cell activation promoted by growth factors. Forty two hours before starting the experiment, cells were cultured in $0.2 \%$ FBS in order to synchronize cells on cell cycle. In order to prepare $\mathrm{CM}$, cell number was normalized by plating the same cell amount of cells in each assayed culture plate for individual OPN-SI overexpressing clone.

Cell migration assays were evaluated by in vitro wound closure and transwell assays, as described by others [15]. Wild-type PC-3 or PC-3 cells transfected with either OPNa, OPNb, OPNc, or empty vector were grown in six-well microtiter plates to near-confluent level in RPMI medium containing 20\% FBS. Mitomycin C ( $5 \mu \mathrm{g} / \mathrm{ml}$; Sigma) was added to inhibit cell proliferation, and the cells were incubated for additional $3 \mathrm{hr}$. Multiple uniform and constant streaks were 
made on the monolayer culture with $10 \mu \mathrm{l}$ pipette tips. Plate dishes were immediately washed with PBS to remove detached cells. Cell migration was monitored for $72 \mathrm{hr}$, and pictures were taken at $0,18,24,48$, and $72 \mathrm{hr}$ time points with a digital camera attached to an inverted Nikon phase contrast microscope.

To determine the effect of OPN isoforms on the invasion potential of PC-3 cells, transwell invasion in vitro assays were performed as reported [16]. Cell culture inserts (24-well, pore size $8 \mu \mathrm{m}$; BD Biosciences) were seeded with 25,000 wild-type PC-3 cells, PC-3 cells transfected either with OPNa, OPNb or OPNc plasmid constructs, or pCR3.1 empty vector in $500 \mu \mathrm{l}$ of medium with $0.1 \%$ FBS. Pre-coated inserts with growth factor reduced Matrigel $(40 \mu \mathrm{l}, 1 \mathrm{mg} / \mathrm{ml}$; BD Biosciences) were used. As chemotactic agent added at the lower chamber, $750 \mu \mathrm{l}$ of CM containing $20 \%$ FBSs has been used. After $48 \mathrm{hr}$, non-invading cells were wiped from the upper side of the membrane and cells on the lower side were fixed in glutharaldeyde followed by staining with $0.1 \%$ crystal violet. The lower side of the filter was photographed by using a phase-contrast microscope at 10-fold magnification and the number of cells was manually counted. Each individual experiment had triplicate inserts and six microscopic fields were counted per insert.

Anchorage independent growth was analyzed in soft agar medium. Six-well plates were coated with $1.2 \%$ agarose-supplemented growth medium to resist cell adhesion. PC-3 wild-type or PC-3 cells transfected with either $\mathrm{OPNa}, \mathrm{OPNb}, \mathrm{OPNc}$, or empty vector were then trypsinized, and $5 \times 10^{2}$ cells were resuspended in $3 \mathrm{ml}$ of growth medium containing $0.6 \%$ agarose. After being plated, colonies were allowed to grow for 4 weeks. Representative colonies were visualized by both phase contrast microscopy and the total number of colonies was determined.

\section{Preparation of Cell Lysates and Immunoblot for Detecting OPN Isoforms}

Cells were harvested and rinsed twice with PBS. Cell extracts were prepared using cell lysis solution containing SDS $1 \%$ and phosphatase and protease inhibitors. Cells were scraped and total protein extract was purified using $20 \mathrm{~mm}$ mini spin Receiver Columns (Macherey-Nagel) by centrifuging 1,400 rpm for $1 \mathrm{~min}$ and then cell extracts collected. Total protein concentration was measured using the BCA assay kit (BioRad), according to the manufacturer's instructions. Immunoblot assays for detecting protein isoforms were performed using $25 \mu \mathrm{g}$ of purified protein extracts, which were subjected to a $7.5 \%$ SDS / PAGE and the resolved proteins were transferred electrophoretically to PVDF (Millipore) membranes. Membrane was blocked for $1 \mathrm{hr}$ using 5\% non-fat dry milk diluted in Tris buffered saline (5\%/TBS). Then, membranes were incubated $\mathrm{O} / \mathrm{N}$ with the human anti-OPNc primary antibody (R \& D systems) diluted 1:500 in 5\%/TBS. After three washes for $10 \mathrm{~min}$ in TBS buffer, membranes were incubated for $1 \mathrm{hr}$ with the anti-goat IgG-HRP secondary antibody. Chemiluminescence detection was performed using ECL PLUS (Amersham Biosciences) in accordance with the manufacturer's instructions. As positive controls for staining the three OPN isoforms with anti-OPNc antibody, we used whole cell extracts from MDA-MB-435 (breast cancer human cell lines) and OPN recombinant protein.

\section{Treatment of Cells With LY294002 and Immunoblotting}

LY294002, a PI3K inhibitor, was obtained from Cell Signaling Technology (Maryland). Untransfected PC-3 cells and those overexpressing either OPN-SI or empty vector control were cultured as described above for cell proliferation, migration, and soft agar colony assays and then treated with $50 \mu \mathrm{M}$ of LY294002. Immunoblot was performed using $50 \mu \mathrm{g}$ of protein extracts. Cells were harvested and rinsed twice with PBS. Cell extracts were prepared with Cell Lysis Buffer (Cell Signaling Technology), sonicated and cleared by centrifugation at $15.000 \mathrm{~g}$. Total protein concentration was measured using the BCA assay kit (BioRad), according to the manufacturer's instructions. PI3Kinase activation was analyzed by the levels of Akt $\mathrm{Ser}^{473}$ phosphorylation. Membranes were incubated with anti-total Akt and anti-phospho-Akt antibodies (Cell Signaling Technology). The horseradish peroxidase (HRP)-conjugated anti-rabbit IgG (Pierce, Rockford, IL) were diluted 1:1,000 in PBST containing 5\% bovine serum albumin. Chemiluminescence detection (Amersham Biosciences) was performed in accordance with the manufacturer's instructions.

\section{In vivoTumorigenicity of OPN-SI Transfectants}

For the analysis of tumor progression in vivo we used 6 weeks athymic BALB/ $\mathrm{c}^{\text {nude/nude }}$ mice. Mice were injected subcutaneously (s.c.) into the left flank with $5 \times 10^{6}$ of PC-3 cell clones. Tumor volumes (V) were analyzed every 2 days using the following formula: $\mathrm{V}\left(\mathrm{mm}^{3}\right)=$ width $\times$ length $^{2} \times 0.52$. A total of 25 mice were randomly assigned to five groups (five mice/group) corresponding to five transfectant clones. Pictures were taken 24 days after injection.

\section{Data and Statistical Analysis}

All results are presented as mean \pm standard error of at least three independent experiments. For in vitro and in vivo data assays, statistical comparisons 
among groups were performed by the Student's $t$-test or ANOVA. $P<0.05$ was considered significant. $P$-values are indicated in the Figure legends.

\section{RESULTS}

\section{Overexpression of OPN-SI in PCa Cells Potentially PromotesTumor Growth and Cell Proliferation}

We previously demonstrated that all OPN-SI are overexpressed in $\mathrm{PCa}$ in relation to $\mathrm{BPH}$ samples and that among these isoforms, OPNc was the most upregulated in PCa samples. Additionally, we also demonstrated that OPNc protein is overexpressed in high Gleason Score (GS) tumor samples, as compared to low GS samples [14]. To further validate these data and compare OPN-SI expression profiling between tumor and non-tumoral prostate samples, we used herein OPN-SI specific oligonucleotides and qRT-PCR to evaluate the expression level of these three splice variants in prostate tumoral and non-tumoral cell lines (Fig. 1A). We found that the three OPN-SI are overexpressed in PCa cell lines, as compared to non-tumoral samples. These data, in association with our previous results [14], indicated that OPN-SI expression levels could be involved in PCa progression. Based on these data, we then assessed the functional significance of elevated OPN-SI expression during the development of $\mathrm{PCa}$ by investigating whether the stable overexpression of each OPN-SI in a human PCa cell line could alter tumor growth in vivo and in vitro.

PC-3 cell line was stably transfected with expression plasmids containing the three OPN-SI. The analysis of isolated clones demonstrated that each OPN-SI overexpression clone present higher mRNA levels of the corresponding isoform in relation their endogenous levels in empty vector transfected cells (Supplementary Fig. 1A). This was further demonstrated at the protein level (Fig. 1B). Immunoblot analysis of total protein
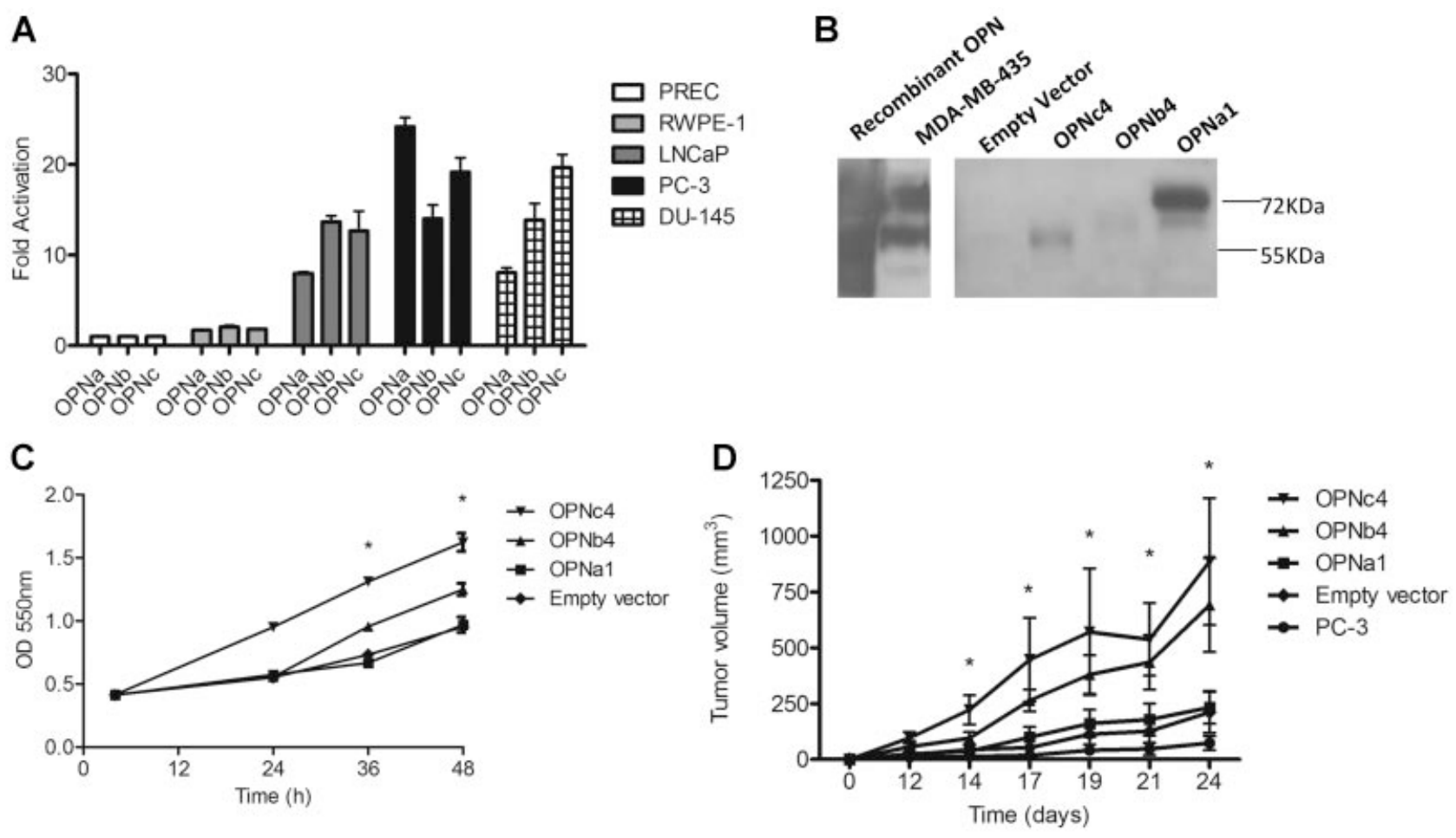

Fig. I. OPN-Sl are overexpressed in PCa and support tumor growth. OPN-Sl expression levels were analyzed by qRT-PCR and were represented by fold activation in relation to the sample presenting the lowest expressing levels. GAPDH was used as a normalization control. A: OPN-SI mRNA expression levels was analyzed in different prostate cell lines. Results are representative of three independent experiments. Horizontal bars represent standard deviations (S.D.). B: Immunoblot analysis using the human anti-OPN antibody and total protein extracts from OPN-SI overexpression clones demonstrating the overexpression of OPNc (around $55 \mathrm{KDa}$ ), OPNb (around $60 \mathrm{KDa}$ ), and OPNa (around $72 \mathrm{KDa}$ ) protein isoforms in each corresponding overexpression clone, respectively. OPN-SI molecular weights presents variability according to post-translational modifications which are cell type dependent. Recombinant OPN and MDA-MB-435 total protein extracts were used as positive controls for OPN-SI detection and image on the right demonstrates the X-ray film exposed in a shorter time to better demonstrate the bands corresponding to the three isoforms. C: PC-3 transfected cells with either OPNa, OPNb or OPNc, or empty vector control and proliferation kinetics analysis was evaluated by crystal violet staining. ${ }^{*} P<0.05$ versus empty vector control clone. $\mathbf{D}$ : Tumor growth rate of mice s.c implanted with OPN-SI overexpression cells and empty vector controls. PC 3 non-transfected cells were also implanted. ${ }^{*} P<0.05$ versus empty vector cells. 
extracts from each OPN-SI isolated clone was probed with a human anti-OPN antibody ( $\mathrm{R} \& \mathrm{D}$ systems), which recognizes three OPN isoforms. This antibody recognizes predominantly OPNc (around $55 \mathrm{KDa}$ ), $\mathrm{OPNb}$ (around $60 \mathrm{KDa}$ ), and $\mathrm{OPNa}$ (around $72 \mathrm{KDa}$ ) isoforms on $\mathrm{OPNc}, \mathrm{OPNb}$, and $\mathrm{OPNa}$ overexpression clones, respectively. These data demonstrated that each overexpression clone present higher levels of the corresponding protein OPN splicing isoform (Fig. 1B). We firstly asked whether the proliferation rates of PC-3 cells overexpressing $\mathrm{OPNa}, \mathrm{OPNb}$, and $\mathrm{OPNc}$ were altered as compared to an empty vector control clone. As shown in Figure 1C, OPNc overexpressing cells significantly activated proliferation rates as compared to $\mathrm{OPNa}$ and empty vector control clone in the range of $4-48 \mathrm{hr}$ of cell culture $(P<0.05)$. $\mathrm{OPNb}$ also stimulated PC-3 cell proliferation, however, presenting no statistical significance $(P=0.07)$, as compared to controls. Similar results were obtained when analyzing proliferation rates by trypan blue assays (Supplementary Fig. 2A). The same proliferation behavior was observed when testing stably expressing clones presenting different OPN isoform expression levels, as demonstrated on Supplementary Fig. 2B-D. To further assess the functional significance of elevated expression of OPN-SI during the

A
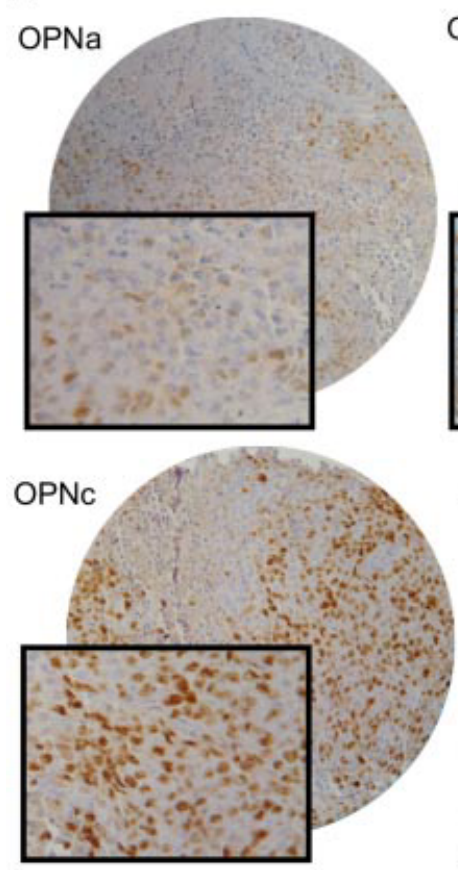
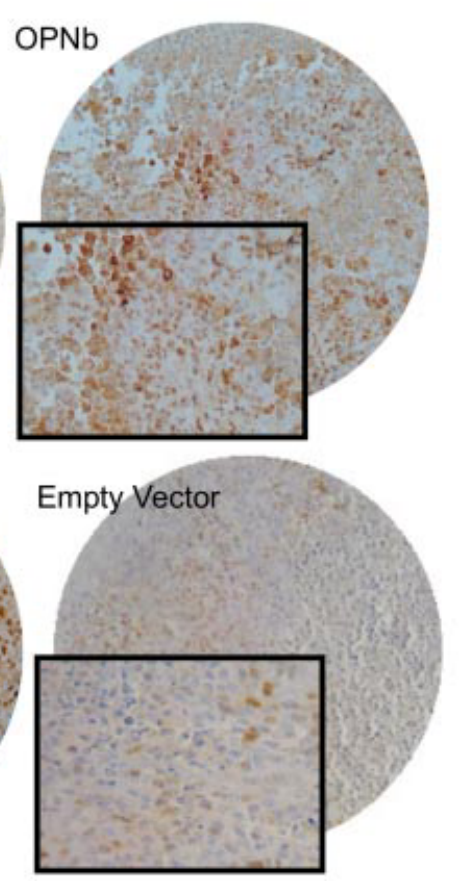

development of $\mathrm{PCa}$, we then investigated whether stable overexpression of each OPN-SI in PC-3 cell line could alter the tumor growth in vivo. Tumors overexpressing OPNc, and to a lesser extent $\mathrm{OPNb}$, grew faster and produced tumors with higher volume as compared to controls (Fig. 1D and Supplementary Fig. 3). Implantation of cells overexpressing OPNc resulted in extremely rapid tumor growth and formation of larger $\left(\geq 500 \mathrm{~mm}^{3}\right)$ tumors between 17 and 19 days after s.c. injection. In contrast, tumors resulting from OPNa or empty vector overexpressing clones were of comparable size $\left(<200 \mathrm{~mm}^{3}\right)$ to those generated with empty vector control cells. Taken together, these results indicated that overexpression of OPNc and $\mathrm{OPNb}$ are able to enhance xenograft prostate tumor growth rates, suggesting that these isoforms accelerate tumor progression.

Tissue sections from xenografts expressing the three OPN-SI constructs were stained for Ki-67 to assess proliferation. As shown in Figure 2A,B, the percentage of $\mathrm{Ki}-67$ positive nuclei in OPNc and $\mathrm{OPNb}$ overexpressing tumors was 3.5 and 2.5-fold higher, as compared to cells overexpressing OPNa and empty vector control, respectively. Ki-67 staining, in addition to cell proliferation in vitro assays (Fig. 1C), indicated that $\mathrm{OPNc}$ and $\mathrm{OPNb}$ overexpression were associated

\section{B}

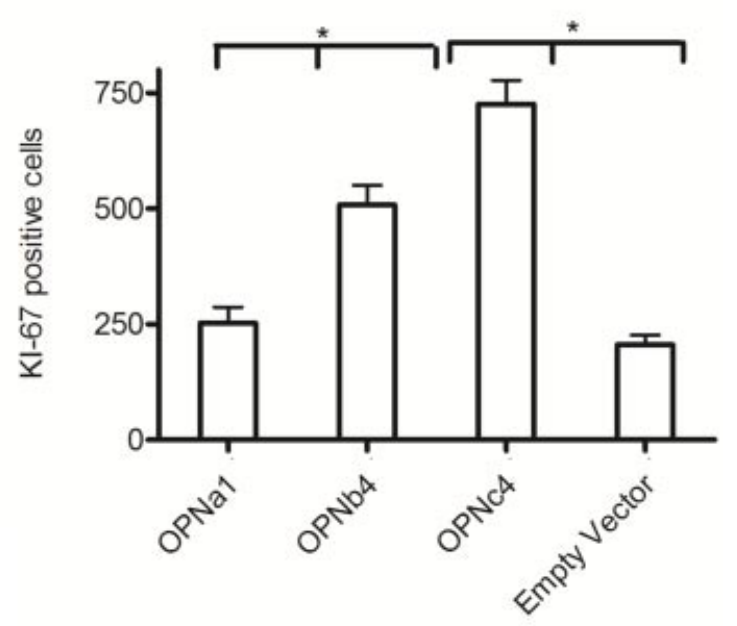

Fig. 2. OPNc and OPNb activate tumor formation by inducing proliferation. Tumors formed by each OPN-SI PC-3 overexpressing cells, as well as empty vector were analyzed for $\mathrm{Ki}-67$ positive staining. A: Representative images of xenograft tumor spots showing Ki- 67 expression as determined by nuclear staining, characterized by a dark brown reaction in the nucleus of tumor cells. $10 \times$ objectives on an Olympus BH-2 microscope with the inset showing a $40 \times$ magnified view of the same field. B: Quantification of immunohistochemical Ki- 67 positive nuclei staining as tested in (A). 
with increased PCa tumor growth. Real time PCR analysis of total RNA obtained from xenograft tumors resulting from $\mathrm{OPNa}, \mathrm{OPNb}$, and $\mathrm{OPNc}$ overexpression clones demonstrated that these tumor tissues maintain each OPN-SI overexpression as compared to tumors formed by empty vector transfected cells (Supplementary Fig. 1B).

To further explore the role of each tumor derived OPN-SI on the regulation of downstream molecules involved on PCa tumor progression, we examined whether tumor xenografts overexpressing OPN-SI could induce MMP-2, MMP-9, and VEGF mRNA expression. In $\mathrm{PCa}$, a functional interplay and an increased expression of these gene products is associated with invasive and metastatic potential. As shown in Figure $3 \mathrm{~A}-\mathrm{C}$, the expression levels of these transcripts were up-regulated in $\mathrm{OPNc}$ and $\mathrm{OPNb}$ overexpressing xenografts, as compared to those tumors resulting from empty vector control $(P<0.01)$.

We then explored the functional mechanisms by which OPN variants promote tumor growth. In order to achieve this objective, we examined the biological effects of OPN-SI overexpression in PC-3 cells under in vitro conditions that mimic important steps of tumor progression.

\section{Secreted OPNc and OPNb Activate Sustained PC-3 Proliferative Signaling}

In serum-starving conditions, cells overexpressing $\mathrm{OPNc}$ and to a lesser extent, OPNb, can significantly enhance cell proliferation (Fig. 4A). These results suggest that OPNc and OPNb modulates the growth of PCa cells independently of growth factors, typical
A

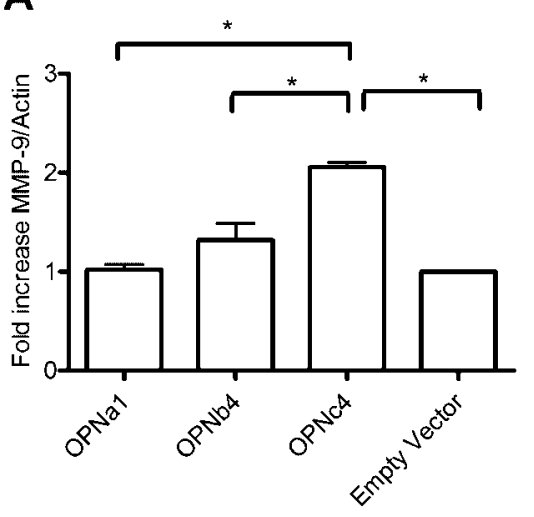

D

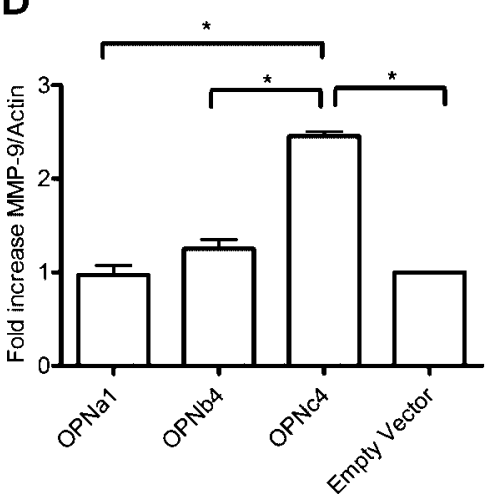

B

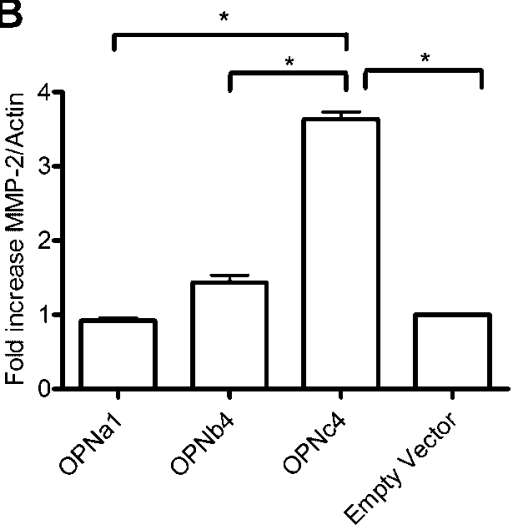

E

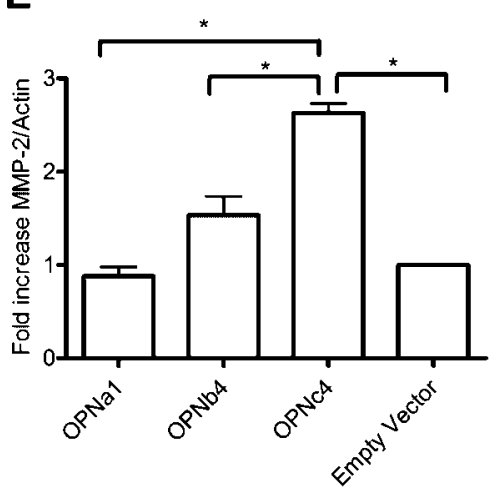

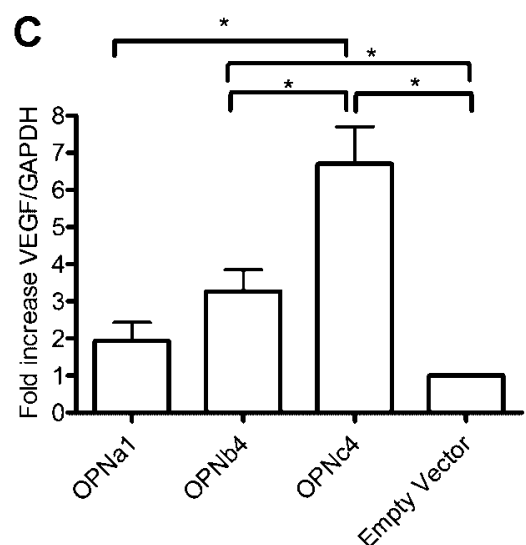

$\mathbf{F}$

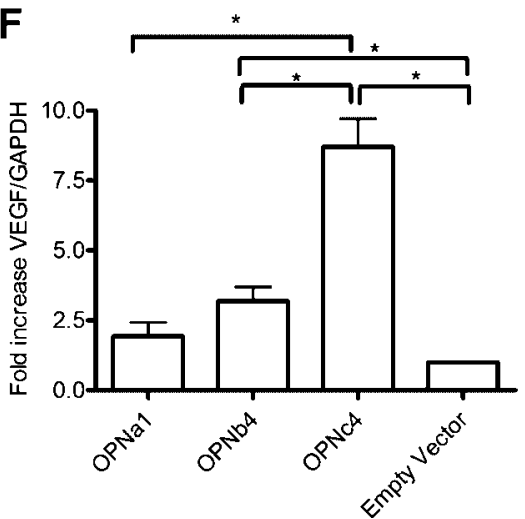

Fig. 3. MMPs and VEGF are overexpressed in PCa cells overexpressing OPNb and OPNc isoforms. A-C: Induction of MMP2 (A), MMP9 (B), and VEGF (C) mRNA expression in xenograft tumors formed by cells overexpressing the three OPN-SI, as compared to tumors formed by PC3 cells transfected with empty vector controls. D-E: Induction of MMP2 (D), MMP9 (E), and VEGF(F) mRNA expression in PC3 cells overexpressing the three OPN-SI as compared to PC3 cells transfected with empty vector controls. Total RNA from cells overexpressing OPNa, OPNb, OPNc, and empty vector control was prepared to conduct quantitative real-time PCR (qRT-PCR) analysis using glyceraldehyde 3-phosphate dehydrogenase (GAPDH) or actin as internal controls. D-E: The amount of targets was analyzed using the comparative CT method, where the threshold cycle (CT) values of each target sequence are given by the 2DDCT formula. We present the data as log $\mathrm{n}$-fold change in gene expression normalized to the endogenous reference genes (GAPDH or actin) relative to the expression of cells overexpressing empty vector control. ${ }^{*} P<0.002$. 


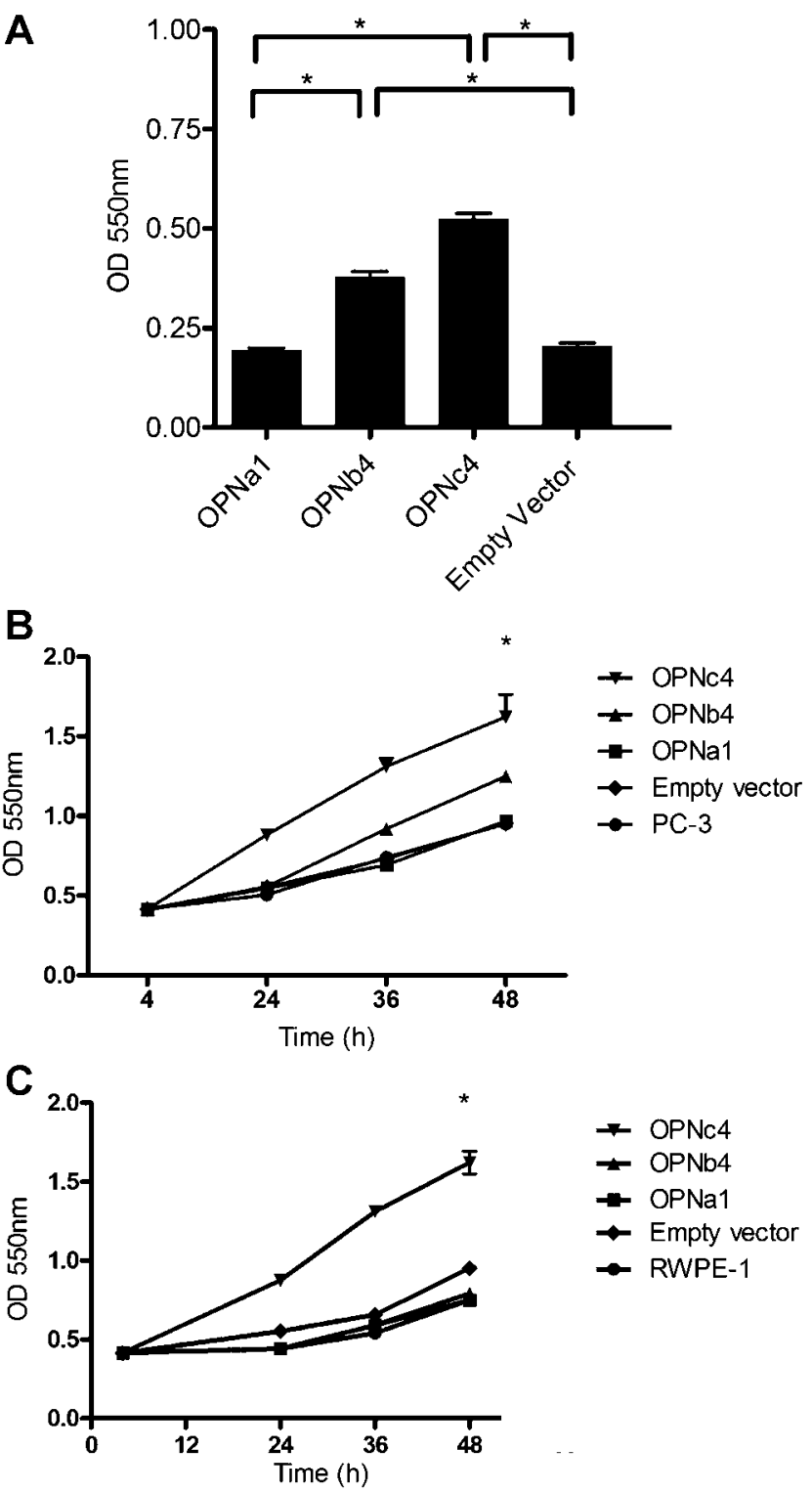

Fig. 4. OPNc and OPNb stimulate cell proliferation under serum starving conditions by stimulating cell survival. A: Cells were grown in $0.2 \% \mathrm{FBS}$ and cell numbers were counted by crystal violet staining at $96 \mathrm{hr}$ after plating. O.D., optical density measured at $550 \mathrm{~nm}$. ${ }^{*} P<0.000$ l versus empty vector control clone. All results are representative of at least three independent experiments. PC-3 (B) and RWPE-I (C) non-transfected cells were assayed for cell proliferation rates by crystal violet staining after incubation with conditioned medium (CM) secreted from OPN-SI and empty vector overexpressing cells. Legend on the right indicate from which cell clones the $C M$ was obtained. Results are representative of at least three independent experiments. O.D., optical density at $550 \mathrm{~nm}$. The standard deviations (error bars) indicate the variability within each experiment. ${ }^{*} P<0.05$ versus $P C-3$ or RWPE-I non-transfected cells.

features of oncoproteins [17]. We then hypothesized that the higher proliferation rates observed for OPNc and $\mathrm{OPNb}$ overexpressing clones could be explained by induction of cell death promoted by $\mathrm{OPNa}$ overexpression. All three OPN-SI cell clones presented similar proportions of cell death, as shown by exclusion of trypan blue analysis (data not shown). These results further evidence the potential role of $\mathrm{OPNc}$ and $\mathrm{OPNb}$ on favoring PCa cell proliferation and survival, which is not associated with stimulated cell death promoted by OPNa or control cells.

We also evaluated proliferation-kinetics of untransfected PC-3 and RWPE-1 cells in the presence of conditioned $\mathrm{CM}$ produced by each OPN-SI and empty vector overexpressing cell lines (Fig. 4B,C). We found that untransfected PC-3 cells cultured in OPNc-CM and $\mathrm{OPNb}-\mathrm{CM}$ displayed higher proliferation rates as compared to controls. Of note, OPNc-CM, but not $\mathrm{OPNb}-\mathrm{CM}$, stimulated the proliferation of RWPE-1 non-tumoral prostate epithelial cells (Fig. 4C). These changes in PC-3 cell proliferation indicates that cell growth were directly dependent on the secreted OPNc protein, as the addition of a polyclonal anti-OPNc antibody, but not control anti-rabbit goat IgG immunoglobulin, significantly suppressed proliferation rates promoted by PC-3 overexpressing OPNc (Supplementary Fig. 4A). Similar results were obtained when performing these assays using an IgY non-specific antibody as a negative control (data not shown). Accordingly, these cells also presented an increase in cell death, providing additional evidence for a survival effect for secreted OPNc in this prostate tumor cell line (Supplementary Fig. 4B). The same effect was not observed when anti-OPNc antibody was added to OPNa overexpression clones (Supplementary Fig. 4C,D). When the same procedure was performed on wild type PC3 non-transfected cells, although presenting no significant effect on cell proliferation, anti-OPNc antibody increased cell death rates in these cells, indicating that even basal OPNc levels in PC3 cells could potentially also exert pro-survival roles (Supplementary Fig. 4E,F). These data indicated that this antibody could specifically inhibit the effect of OPNc protein isoform on cell proliferation and survival. Altogether, these data indicated that secreted OPNc, in addition to enhancing proliferation of PCa cells, also stimulates proliferation of normal prostate epithelial cells, further suggesting a role of this isoform in prostate tumorigenesis and cell survival. Conversely, the $\mathrm{OPNb}$ isoform likely exerts its main effects on cell proliferation at later stages of $\mathrm{PCa}$ progression. A serial dilution analysis of OPNc and OPNb-CM promoted a proportional decrease on PC3 cell proliferation (Supplementary Fig. 5A,C). On the other hand, OPNb-CM serial dilution analysis only promoted a proportional decrease on PC3 cell proliferation (Supplementary Fig. 5B). On RWPE1 cells, proliferation rates were maintained, even according to $\mathrm{OPNb} \mathrm{CM}$ serial dilution analysis (Supplementary Fig. 5D). 


\section{PC-3 Cells Overexpressing OPNc and OPNb Stimulate Prometastatic Processes}

We then elucidated whether OPN-SI overexpressed in PC-3 cells are able to induce altered phenotypes associated with additional processes favoring tumor progression, such as migration, invasion, and anchorage independent cell growth.

PC-3 cells overexpressing OPN-SI or empty vector were subjected to an in vitro wound closure migration assay (Fig. 5A). Migration of OPNc and OPNb expressing cells were enhanced as compared to OPNa and empty vector-expressing cells. Eighteen hours after the scratch assay was initiated, OPNc and OPNb enhanced PC-3 cell motility into the wound. After $24 \mathrm{hr}$, $\mathrm{OPNc}$ and $\mathrm{OPNb}$ overexpressing cells completely closed the wound edges (data not shown).

$\mathrm{OPNc}$ and $\mathrm{OPNb}$ overexpression also stimulated PC-3 invasion capacity by at least 5.0-fold and 2.5fold ( $P<0.0001)$, as compared to cells overexpressing OPNa and empty vector control (Fig. 5B). These results suggest that OPNc and OPNb isoforms increased cell migration and invasion of PC-3 cells in vitro.

\section{A}
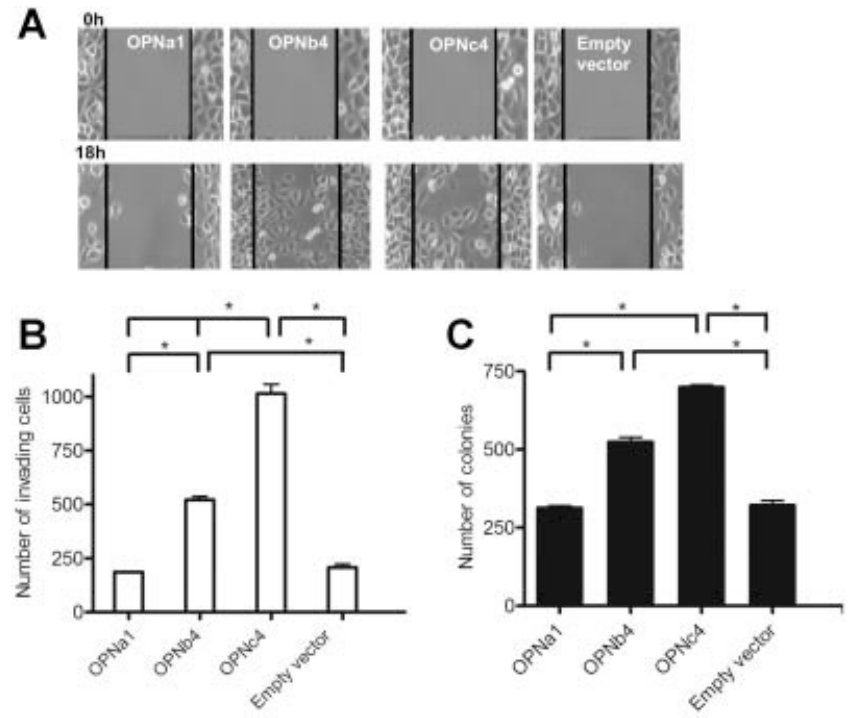

Fig. 5. OPNc and OPNb stimulate prometastatic processes in PCa cells. Cell clones of OPN-SI and empty vector control cells were analyzed for cell migration (A), invasion (B), and anchorage independent growth (C). A: Phase-contrast micrographs photographs were taken at 0 and $18 \mathrm{hr}$ after clones subjected to migration by wound closure assays are shown. Samples were tested in triplicates. B: Invading cells tested by transwell invasion assays were stained with crystal violet and the number of cells manually counted. Data are reported as average of number of invading cells \pm S.D. of three independent experiments. ${ }^{*} P<0.001$. C: Quantification of the number of PC-3 cell colonies grown in semisolid agarose medium transfected with different constructs, as indicated. Results are representative of at least three independent experiments. ${ }^{*} P<0.01$ vesrsus empty vector cells.
Similarly to in vivo assays (Fig. 3A-C), there was a significant increase in MMP-2, MMP-9, and VEGF mRNA levels $(P<0.01)$ in $P C-3$ cells overexpressing $\mathrm{OPNc}$ and $\mathrm{OPNb}$, as compared to OPNa and empty vector overexpressing clones (Fig. 3D-F). These results indicated that the upregulation of these transcripts could be one of the molecular mechanisms by which these OPN variants can activate PC-3 cell migration and invasion capacity. OPNc-CM and $\mathrm{OPNb}-$ CM also significantly activated PC-3 cell migration and invasion (data not shown), reinforcing the notion that the secreted isoforms of these OPN variants acts as stimulatory factors for PC-3 cell proliferation, migration, invasion, and metastatic potential.

To examine whether OPN-SI overexpression also alters the ability for anchorage-independent growth as a measure for tumorigenic potential, soft agar assays were performed. OPNc and $\mathrm{OPNb}$ overexpressing PC-3 cells significantly increased the number of colonies formed, while OPNa isoform promoted no significant effects (Fig. 5C). The three OPN-SI overexpression clones also promoted an increase in the size of colonies formed, although OPNc and $\mathrm{OPNb}$ displayed a more pronounced effect (data not shown). These data suggest that OPNc and OPNb can act as activating factors to enhance anchorage independent growth, indicative of their roles on regulating metastatic potential.

\section{OPNc and OPNb Mediate PCa Progression Features Through the PI3K/Akt Pathway}

During PCa progression, tumor cells undergo molecular alterations that lead to the acquisition of uncontrolled growth properties. One such set of molecular alterations may be mediated by the PI3K-Akt signaling pathway [18]. To explore the molecular mechanisms by which OPN-SI mediate their effects on PCa progression, we examined if the overexpression of each OPN-SI would modulate PI3K activity and Akt phosphorylation $\left(\operatorname{Ser}^{473}\right)$.

As shown in Figure 6A, an increased phosphorylation level on Ser $^{473}$ was readily detected in protein extracts of OPNc and $\mathrm{OPNb}$ overexpression cells (2.1-fold and 1.46-fold increase, respectively), but not in the vector-controls or OPNa overexpressing cells. Differential ERK 1/2 hyperphosphorylation was not observed among cell extracts overexpressing all OPNSI (data not shown). The activation of PC-3 cell proliferation, migration and soft agar colony formation mediated by OPNc and OPNb overexpression were significantly inhibited when these cells were treated with LY294002 (Fig. 6B-D). PC-3 cells overexpressing $\mathrm{OPNc}$ and $\mathrm{OPNb}$ could better overcome the effects of LY29400 on inhibiting the number of soft agar 
A

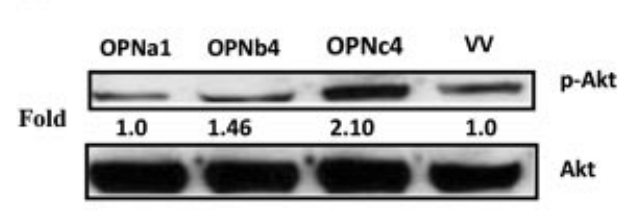

B

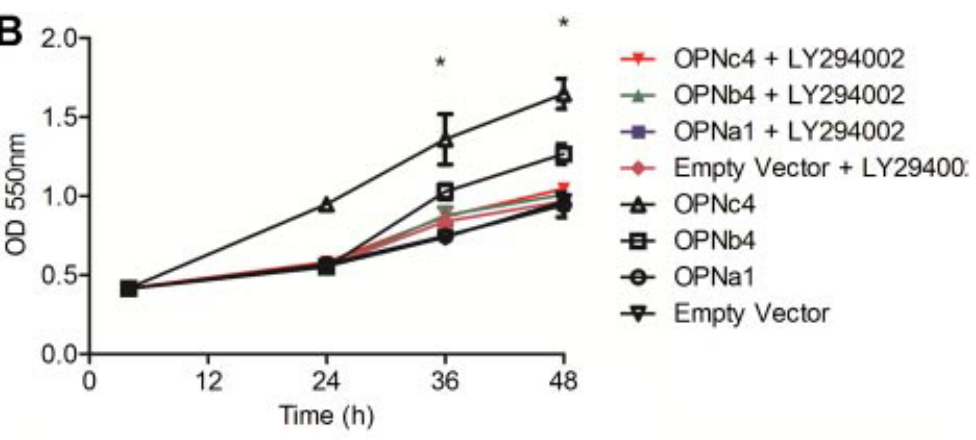

C
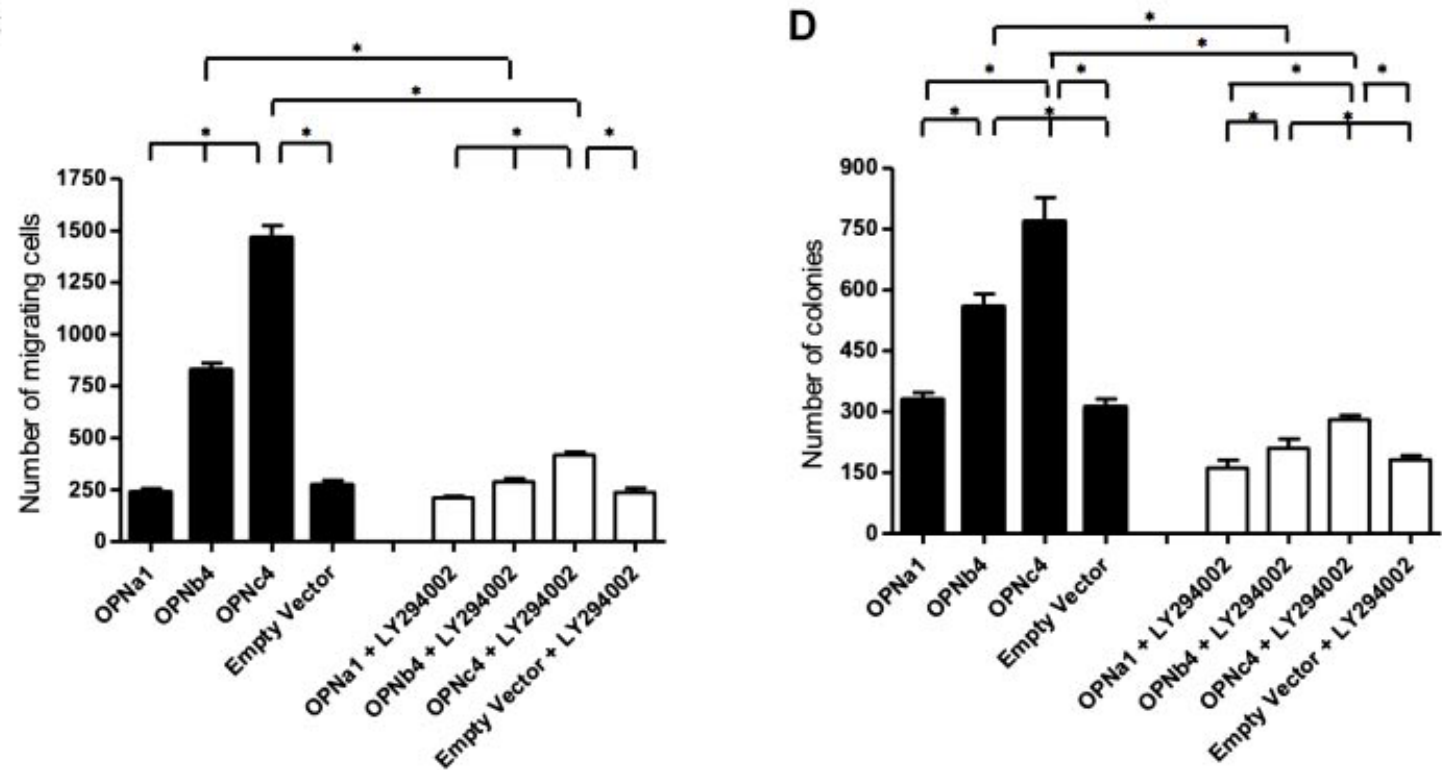

Fig. 6. $\mathrm{PI} 3 \mathrm{~K} / \mathrm{Akt}$ signaling pathway mediate $\mathrm{ONPc}$ and $\mathrm{OPNb}$ roles on activating tumor progression features. A: Total Akt and $\mathrm{p}-\mathrm{Akt}$ $(60 \mathrm{KDa})$ levels in PC-3 cells overexpressing OPN-Sl and empty vector control were measured by immunoblot using their specific antibodies. Non-phospho Akt was used as a loading control. Fold changes were calculated based on densitometric quantification. B: Growth curves of PC-3 overexpressing cell clones OPNc, OPNb, OPNa, and empty vectors cultured with or without LY294002 are plotted, as indicated at the legend on the right. ${ }^{*} P<0.05$ vesrsus empty vector control clone. C PC-3 cells overexpressing each OPN-SI, as compared to empty vector control were evaluated for cells migration capacity by transwell assays after LY294002 treatment at the $48 \mathrm{hr}$ time point. D: The number colonies of anchorage independent growth formed by PC-3 cells overexpressing each OPN-Sl as compared to empty vector control cells was analyzed in the presence or absence of LY294002 inhibitor. ${ }^{*} P<0.002$.

colonies formed (Fig. 6D). These results indicate that PI3K signaling is mediating tumor progression features evoked by OPNc and OPNb overexpression.

\section{DISCUSSION}

Current data debate that OPN splice variants present different tumor and tissue specific roles. While in some tumor models an individual OPN-SI behaves as a tumor stimulating factor, in other tumor contexts the same variant presents just the opposite effect, acting individually or in concert with the remaining isoforms to modulate tumor progression [8-11,13]. Although total OPN has been a key biomarker for detecting PCa progression, no reports until now have described the roles of each OPN-SI in prostate tumorigenesis [4-7]. Herein, we attempted to characterize the involvement of each OPN-SI as an important approach to understand some of the aberrant and deregulated genetic control in PCa.

We previously demonstrated that OPN-SI are overexpressed in PCa tissues in relation to $\mathrm{BPH}$ samples and that OPNc protein was up-regulated in high gleason score tumor samples [14]. Data provided herein reinforced OPN-SI transcript overexpression in PCa cell lines as compared to non-tumoral ones. Based on this, we then hypothesized whether this OPN-SI overexpression in PCa could play important role in PCa progression.

We found that cells overexpressing OPNc and $\mathrm{OPNb}$, but not the OPNa splice variant, are able to enhance PCa tumor growth in vivo, mainly mediated 
by inducing cell proliferation. In vitro, although $\mathrm{OPNb}$ overexpressing cells promoted an increase at proliferation rates, the results were not significant. These controversial findings indicate that the role of $\mathrm{OPNb}$ on activating tumor growth and cell proliferation in vivo could be potentially mediated by paracrine signals secreted by the tumor microenvironment, as has been intensely investigated $[19,20]$. It remains to be determined whether $\mathrm{OPNb}$ acts independently or in concert with OPNc to activate signaling pathways that promote $\mathrm{PCa}$ progression.

In serum starvation conditions, we showed that cell overexpressing $\mathrm{OPNc}$, and to a lesser extent $\mathrm{OPNb}$, still enhanced PC-3 proliferation. These data indicated that OPN-SI can promote tumor progression in a manner that is independent of growth factors, a typical feature of oncogenic gene products [17]. The data presented here provide evidence that cells overexpressing OPNc and OPNb have enhanced cell proliferation due to the promotion of cell survival by these isoforms. First, cells overexpressing OPNc and OPNb stimulated PI3K pro-survival signaling, which could be one of the molecular mechanisms of activating pathways activating cell proliferation and/or rescuing cells from apoptotic stimuli, such as serum starvation [18,21]. Second, PC-3 cells overexpressing OPNc had decreased proliferation and were induced to die, when treated with an anti-OPNc antibody. Finally, cells overexpressing OPNc and OPNb treated with LY294002 had improved survival, overcoming apoptosis typically induced by this drug treatment. Previous reports found that total OPN is able to prevent cell death in response to diverse stress stimuli, including serum starvation and also inhibit apoptosis in several systems [21], but no reports have described previously the individual role of each OPN-SI. Based on published reports and our present results, we propose that OPNc and OPNb splicing isoforms may enhance PC-3 survival due to enhanced cell proliferation. Further studies should be carried out to determine the cellular and mechanisms able to stimulate cell growth evoked by OPNb and OPNc overexpression and whether apoptosis or other mechanisms of cell death inhibition are also involved in mediating the survival roles for $\mathrm{OPNc}$ and $\mathrm{OPNb}$ in PCa. Besides promoting higher proliferating rates in PC-3 tumor cells, we have also showed that secreted OPNc also stimulates RWPE-1 (a normal prostate epithelia cell line) proliferation. Therefore, these data indicated that OPNc is not only involved in PCa progression and survival, but may also contribute to early steps of $\mathrm{PC}$ a tumorigenesis. OPNb, on the other hand, appears to exert its main effects on cell proliferation at later stages of tumor progression. Our data also demonstrated that OPNc and OPNb stimulated PC-3 cell motility and invasion and soft agar colony formation, inferring the roles of these isoforms on metastatic potential. Corroborating to these observations, cells overexpressing OPNc and OPNb isoforms significantly activated in vitro and in vivo the expression of MMP2, MMP-9, and VEGF, molecules critical to PCa invasion and metastatic potential [22]. Some reports detailed the signaling pathways mediating each one of the OPN-SI roles. For instance, in hepatocarcinoma cell lines, OPN-SI differentially activated migrationassociated signaling pathways. OPNa and $\mathrm{OPNb}$, but not OPNc, increased the expression of urokinase type plasminogen activator and the phosphorylation of p42/p44 MAP kinase [11]. These same isoforms promoted pancreatic and fibrosarcoma tumors mainly by protecting cells against stress-induced apoptosis, by inducing NF-kappaB activation and FAK phosphorylation [13]. Our group recently demonstrated that OPNc activated ovarian tumor progression features by inducing the PI3K signaling pathway [8]. The present work adds a mechanistic understanding of the roles of OPN-SI in tumor progression by demonstrating that both OPNc and OPNb can activate distinct aspects of PCa progression through activation of PI3K signaling. Hence, specific knowledge of which signaling pathway and specific OPN-SI are mediating tumor progression opens possibilities to apply antisplicing isoforms strategies in addition to signaling pathways inhibitors as therapeutic approaches to inhibit tumor progression [12,23]. Therefore, we propose that OPNc and OPNb-mediated roles on activating PCa progression features may be linked to the activation of PI3K signaling to promote PCa progression in both localized and advanced PCa. In support of our findings, previous studies have demonstrated that treatment of PCa cells with LY294002 resulted in cell cycle-mediated arrest, induction of apoptosis and inhibition on cellular invasion [24,25]. Although LY294002 can inhibit other gene products [26], in our study, PC-3 proliferation and migration capacity were specifically inhibited in OPNc and OPNb overexpressing cells, indicating that at least the effect on these cell features are mainly mediated by PI3K signaling and upstream signals activated by these splicing isoforms. However, cells overexpressing $\mathrm{OPNc}$ and $\mathrm{OPNb}$ overcame soft agar colony formation inhibition, indicating that these OPN-SI could be inducing PI3K signaling, which is classically involved on mediating soft colony formation, cell survival and anoikis, as described [24-26]. Moreover, PI3K/Akt signals have been reported to directly involve the upregulation of MMP-2 and MMP-9 [22,27]. Therefore, it would appear from the present data, that the OPNc and OPNb-mediated signals could possibly operate through a cell receptor/PI3K/Akt/MMP-2/-9 
pathway. Further experiments will focus on verifying which specific receptor and downstream signals are being activated by OPNc and $\mathrm{OPNb}$ to modulate PI3K signaling and promote PCa progression.

\section{CONCLUSIONS}

We provide strong evidence that overexpression of $\mathrm{OPNc}$ and $\mathrm{OPNb}$ splice variants may be involved in distinct steps of PCa progression. Additionally, we demonstrated that the secreted forms of these OPN-SI are mainly mediating OPN effects on tumor progression by activating at least the PI3K pro-survival pathway. Therefore, a better understanding of the molecular mechanisms by which OPNc and OPNb activate PI3K signaling and its main targets will open up possibilities for developing improved PCa therapeutic approaches that specifically downregulate these isoforms and their effects, inhibiting PCa progression.

\section{ACKNOWLEDGMENTS}

We thank Dr. Marxa L. Figueiredo (Department of Pharmacology and Toxicology, University of Texas Medical Branch) and Luciana Bueno Ferreira for reviewing this manuscript.

\section{REFERENCES}

1. Anborgh PH, Mutrie JC, Tuck AB, Chambers AF. Pre- and post-translational regulation of osteopontin in cancer. J Cell Commun Signal 2011;5(2):111-122.

2. Bellahcene A, Castronovo V, Ogbureke KU, Fisher LW, Fedarko NS. Small integrin-binding ligand N-linked glycoproteins (SIBLINGs): Multifunctional proteins in cancer. Nat Rev Cancer 2008;8(3):212-226.

3. Rangaswami H, Bulbule A, Kundu GC. Osteopontin: Role in cell signaling and cancer progression. Trends Cell Biol 2006; 16(2):79-87.

4. Shevde LA, Das S, Clark DW, Samant RS. Osteopontin: An effector and an effect of tumor metastasis. Curr Mol Med 2010;10(1):71-81.

5. Zhang Y, Forootan SS, Kamalian L, Bao ZZ, Malki MI, Foster CS, Ke Y. Suppressing tumourigenicity of prostate cancer cells by inhibiting osteopontin expression. Int J Oncol 2011;38(4): 1083-1091.

6. Robertson BW, Bonsal L, Chellaiah MA. Regulation of Erk1/2 activation by osteopontin in PC3 human prostate cancer cells. Mol Cancer 2010;9:260.

7. Weber GF, Lett GS, Haubein NC. Categorical meta-analysis of Osteopontin as a clinical cancer marker. Oncol Rep 2011;25(2): 433-441.

8. Tilli TM, Franco VF, Robbs BK, Wanderley JL, da Silva FR, de Mello KD, Viola JP, Weber GF, Gimba ER. Osteopontin-c splicing isoform contributes to ovarian cancer progression. Mol Cancer Res 2011;9(3):280-293.

9. He B, Mirza M, Weber GF. An osteopontin splice variant induces anchorage independence in human breast cancer cells. Oncogene 2006;25(15):2192-2202.
10. Blasberg JD, Goparaju CM, Pass HI, Donington JS. Lung cancer osteopontin isoforms exhibit angiogenic functional heterogeneity. J Thorac Cardiovasc Surg 2010;139(6):1587-1593.

11. Chae S, Jun HO, Lee EG, Yang SJ, Lee DC, Jung JK, Park KC, Yeom YI, Kim KW. Osteopontin splice variants differentially modulate the migratory activity of hepatocellular carcinoma cell lines. Int J Oncol 2009;35(6):1409-1416.

12. Bauman JA, Kole R. Modulation of RNA splicing as a potential treatment for cancer. Bioeng Bugs 2011;2(3):125-128.

13. Courter D, Cao H, Kwok S, Kong C, Banh A, Kuo P, Bouley DM, Vice C, Brustugun OT, Denko NC, Koong AC, Giaccia A, Le QT. The RGD domain of human osteopontin promotes tumor growth and metastasis through activation of survival pathways. PLoS ONE 2010;5(3):e9633.

14. Tilli TM, Thuler LC, Matos AR, Coutinho-Camillo CM, Soares FA, da Silva EA, Neves AF, Goulart LR, Gimba ER. Expression analysis of osteopontin mRNA splice variants in prostate cancer and benign prostatic hyperplasia. Exp Mol Pathol 2011;92:13-19.

15. Nishio T, Kawaguchi S, Yamamoto M, Iseda T, Kawasaki T, Hase T. Tenascin-C regulates proliferation and migration of cultured astrocytes in a scratch wound assay. Neuroscience 2005;132(1):87-102.

16. Klausen C, Leung PC, Auersperg N. Cell motility and spreading are suppressed by HOXA4 in ovarian cancer cells: Possible involvement of beta1 integrin. Mol Cancer Res 2009;7(9): 1425-1437.

17. Hanahan D, Weinberg RA. Hallmarks of cancer: The next generation. Cell 2011;144(5):646-674.

18. Shukla S, Maclennan GT, Hartman DJ, Fu P, Resnick MI, Gupta S. Activation of PI3K-Akt signaling pathway promotes prostate cancer cell invasion. Int J Cancer 2007;121(7):1424-1432.

19. Risbridger GP, Taylor RA. Minireview: Regulation of prostatic stem cells by stromal niche in health and disease. Endocrinology 2008;149(9):4303-4306.

20. Zou C, Song G, Luo Q, Yuan L, Yang L. Mesenchymal stem cells require integrin beta1 for directed migration induced by osteopontin in vitro. In vitro Cell Dev Biol Anim 2011;47(3): 241-250.

21. Chinni SR, Sarkar FH. Akt inactivation is a key event in indole3 -carbinol-induced apoptosis in PC-3 cells. Clin Cancer Res 2002;8(4):1228-1236.

22. Castellano G, Malaponte G, Mazzarino MC, Figini M, Marchese F, Gangemi P, Travali S, Stivala F, Canevari S, Libra M. Activation of the osteopontin/matrix metalloproteinase-9 pathway correlates with prostate cancer progression. Clin Cancer Res 2008;14(22):7470-7480.

23. Chatterjee SK, Zetter BR. Cancer biomarkers: Knowing the present and predicting the future. Future Oncol 2005;1(1): 37-50.

24. Ciraolo E, Morello F, Hirsch E. Present and future of PI3K pathway inhibition in cancer: Perspectives and limitations. Curr Med Chem 2011;18(18):2674-2685.

25. Gao N, Zhang Z, Jiang BH, Shi X. Role of PI3K/AKT/mTOR signaling in the cell cycle progression of human prostate cancer. Biochem Biophys Res Commun 2003;310(4):1124-1132.

26. Kuppumbatti YS, Rexer B, Nakajo S, Nakaya K, Mira-y-Lopez R. CRBP suppresses breast cancer cell survival and anchorageindependent growth. Oncogene 2001;20(50):7413-7419.

27. Morgan TM, Koreckij TD, Corey E. Targeted therapy for advanced prostate cancer: Inhibition of the PI3K/Akt/mTOR pathway. Curr Cancer Drug Targets 2009;9(2):237-249. 